\title{
RESUMO DE ARTIGOS
}

LUIS CLAUDIO PANDINI

PANDINI, LC. Resumo de Artigos. Rev bras Coloproct, 2011;30(4): 466-467.

Durães, L C; Sousa, J B. Câncer anal e doenças sexualmente transmissíveis: Qual a correlação?. Ver. Col. Brás. Cir.2010; 37(4); 265-268.

O objetivo deste trabalho foi verificar a correlação entre o câncer Anal e as Doenças Sexualmente Transmissíveis, como HPV,HIV, infecção Gonocócica, infecção por Clamídia, Sífilis e outras. Foram pesquisadas no site do DATASUS as internações por Câncer Anal, HPV, HIV, infecção Gonocócica, Infecção por Clamídia, Sífilis e outras DSTs, no SUS no Brasil, entre 1998 e 2007. O teste de correlação de Pearson foi aplicado. Há uma correlação positiva muito alta entre as internações por Câncer Anal e HPV ( $r=0,98$, $p<0,001)$. Há uma correlação negativa entre as internações por Câncer Anal e as internações por infecções Gonocócica ( $r=0,81, p=0,005)$ e infecção por Clamídia $(r=0,74, p=0,014)$. Não houve correlação estatisticamente significante entre Câncer Anal e as internações por HIV $(r=0,40, p=0,25)$, outras DSTs $(\mathrm{r}=0,55, \mathrm{p}=0,1)$ e Sífilis $\mathrm{r}=0,61, \mathrm{p}=0,059)$. Os autores concluem que há uma correlação positiva muito alta entre as internações por Câncer Anal e HPV no Brasil. Há uma correlação negativa entre as internações por Câncer Anal,infecções Gonocócica e infecção por Clamídia.

Barmparas, G; Branco, B C; Schnuriger, et al. The Incidence and Risk Factors of Post-Laparotomy Adheive Small Bowel Obstruction. Journal of Gastrointestinal Surgery 2010, vol:14; 1619-1628.

O objetivo desta revisão foi avaliar a incidência e fatores de risco para obstrução do intestino delgado por aderências (O I). Um total de 446.331 operações abdominais foram elegíveis para inclusão na análise. A incidência global de OI foi $4,6 \%$. O risco de OI foi altamente influenciada pelo tipo de procedimento anastomose com bolsa íleo-anal esteve associada com a maior incidência de O I(1.018 de 5.266 casos, ou 19,3\%), seguido por colectomia aberta (11491 de 121.085 casos, ou 9,5\%). Procedimentos ginecológicos foram associados com uma incidência global de 11,1\% (4.297 de 38.751 casos). A técnica do procedimento (aberta versus laparoscópica) também desempenhou um papel importante no desenvolvimento da O I por aderências. A incidência foi de 7,1\% em colecistectomia abertas versus $0,2 \%$. Não houve diferença em obstrução intestinal com apendicectomia laparoscópica ou aberta $(1,4 \%$ vs $1,3 \%)$. Não há provas suficientes sobre o papel da idade, sexo e presença de câncer na formação de aderências. Morbidade relacionada a aderências abdominais compreende uma carga significativa de recursos da saúde e a prevenção é de grande importância, especialmente em pacientes de alto risco. Técnicas preventivas e barreiras protetoras especiais devem ser consideradas em casos de alto risco.

Nash, G M.; Weiss, A; Dasgupta. et al. Close distal Nargin and Rectal Câncer Recurrence After Sphincter - Preserving Rectal Resection. Diseases of the Colon \& Rectum, 2010: vol 53; n $101365-1373$.

O propósito deste estudo retrospectivo foi analisar a associação de recorrência do câncer do reto baixo com margem distal restrita ou comprometida. Foram incluídas 627 pacientes submetidos à ressecção curativa baixa anterior com excisão total do mesorreto para o câncer retal $2-12 \mathrm{~cm}$ da borda anal. Trezentos e noventa e nove pacientes receberam tratamento 
neoadjuvante, 65 receberam a terapia adjuvante pós operatória e 163 foram tratadas apenas com cirurgia. O seguimento mediano foi de 5,8 anos. A recidiva total foi associada com o estádio patológico, Invasão linfática e margem distal. Recorrência na mucosa foi incomum; apenas 16 ocorrências foram registradas, e destas apenas oito estavam no local inicial de recorrência do tumor isolado, 7 dos 8 pacientes foram resgatados cirurgicamente. $\mathrm{Na}$ análise univariada, a recorrência da mucosa foi associada com a margem distal estreita ( 5 vs $2 \%$ ) e invasão linfática ( 7 vs $2 \%$ ). Recorrência pélvica sem associação com recidiva da mucosa esteve associada à localização distal (6 contra 4\%) e invasão linfática (11 vs 4\%). Os autores concluem que margem distal comprometida ou restrita identifica pacientes com risco aumentado de recorrência do câncer e da mucosa retal. Embora nem causalidade, nem comprimento das margens minimamente aceitável possa ser definido, os dados reforçam a importância de alcançar uma clara margem de ressecção distal no tratamento cirúrgico do câncer retal.

Kang, SB.;Park,J S.; Kim,DW.;Lee, TG.Intraoperative Technical Difficulty Douring Laparoscopy - Assisted Surgery as a Prognostic Factor for Colorectal Câncer. Diseases of the Colon \& Rectum. 2010 Vol: 53: $n^{\circ} 10$, 1400-1408.

Objetivo deste estudo foi investigar se a dificuldade técnica encontrada durante a cirurgia laparoscópica pode ser considerada um fator de recorrência relacionado ao câncer colorretal. Os dados de 427 pacientes submetidos a cirurgia laparoscópica para câncer colorretal entre maio de 2003 e dezembro de 2007 foram analisados. Uma dificuldade técnica intra-operatória foi definida como um desvio significativo do procedimento cirúrgico padrão. Todas as conversões para cirurgia aberta ou perfuração intestinal iatrogênica durante a cirurgia laparoscópica foram incluídos como dificuldades técnicas. O modelo de Cox de regressão de riscos proporcionais foi utilizado para avaliar o fa- tor de recorrência relacionadas com vários fatores de riscos, incluindo dificuldade técnica. As dificuldades técnicas foram encontradas em $44(10,3 \%)$ pacientes, sendo $17(3,9 \%)$ conversões para cirurgia aberta a 10 $(2,4 \%)$ com lesão iatrogência do intestino. As dificuldades técnicas foram encontradas com mais freqüência em homens comparados com as mulheres $(13,5 \%$ vs $6,0 \%, \mathrm{P}=013)$ e de tumores localizados no reto médio e baixo, flexura esplênica e colon descendente. As taxas de recidiva foram maiores em pacientes com dificuldades técnicas (recidiva local $2,6 \%$ vs $6,7 \% \mathrm{P}$ $<0,05$ ); recidiva sistêmica, $6,3 \%$ vs $13.6 \% \mathrm{P}<0,05$ ), com uma média de seguimento de 45,9 meses. A análise multivariada pelo modelo de regressão proporcional de Cox mostrou que a dificuldade técnica foi um fator de risco independente relacionado à recidiva após a cirurgia laparoscópica (odds ratio, 2,374, 95\% CI, 1,005-5,600, $\mathrm{P}=0,048$ ). A conclusão deste estudo demonstrou que a dificuldade técnica durante a cirurgia laparoscópica assistida compromete a segurança oncológica. Sugere-se que os cirurgiões devem estar preparados para minimizar as dificuldades técnicas durante a cirurgia laparoscópica.

Soltan, A; Kaise, A M. Endorectal Advancement flap for Cryptoglandular or Crohn's fistula-in-Ano. Diseases of the Colon \& Rectum. 2010. Vol: 53: $n^{\circ} 4$ 486-495.

Este estudo de revisão sistemática da literatura foi realizada para avaliar o papel da técnica de retalho mucoso para fistulas anal transesfincterianas complexas criptoglandulares e por doença de Crohn. Fístulas retovaginais e por câncer foram excluídas. Cada estudo foi analisado tempo de seguimento, taxa de sucesso e incontinência anal. Foram analisados 35 estudos com 2065 pacientes sendo 1.654 pacientes com fistulas criptoglandulares ou por Doença de Crohn. A qualidade dos trabalhos foi limitada com nível baixo de evidencia. A taxa de sucesso e incontinência foram $80.8 \%$ e $13.22 \%$ para fistula criptoglandular e $64 \%$ e 9.4\% para fistulas por Doneça de Crohn. 